\title{
BMJ Open Therapy-dependent inconsistencies in self-reported use of complementary and alternative medicine in the general population: findings from a longitudinal study
}

\author{
Heidi Amalie Rosendahl Jensen (D), Ola Ekholm (D)
}

To cite: Jensen HAR,

Ekholm 0. Therapydependent inconsistencies in self-reported use of complementary and alternative medicine in the general population: findings from a longitudinal study. BMJ Open 2021;11:e051647. doi:10.1136/ bmjopen-2021-051647

- Prepublication history for this paper is available online. To view these files, please visit the journal online (http://dx.doi. org/10.1136/bmjopen-2021051647).

Received 25 March 2021 Accepted 05 November 2021

D) Check for updates

(c) Author(s) (or their employer(s)) 2021. Re-use permitted under CC BY-NC. No commercial re-use. See rights and permissions. Published by BMJ.

National Institute of Public Health, University of Southern Denmark, Copenhagen, Denmark

Correspondence to Mr 0la Ekholm; oek@sdu.dk

\section{ABSTRACT}

Objective Information on the use of complementary and alternative medicine (CAM) in the general population is often collected by means of surveys, causing the reliability of data to rely on the memory accuracy of the respondent. The objective of this study was to examine the consistency in self-reported CAM use using data from two survey waves 4 years apart.

Design Longitudinal study.

Setting/participants Data were obtained from the Danish Health and Morbidity Surveys. A nationally representative subsample of the individuals invited in 2013 was reinvited in 2017. In all, 2297 individuals ( $\geq 16$ years) completed the self-administered questionnaire in both waves, including questions on for example, CAM use.

Main outcome measures The use of six different CAM therapies (acupuncture; craniosacral therapy; faith healing and/or clairvoyance; nutritional counselling; massage; osteopathy or other manipulative therapies; reflexology) was assessed by the response categories 'Yes, within the past 12 months', 'Yes, but previously than within the past 12 months' and 'No'. For each CAM therapy, an inconsistent response was defined as either the response combination (1) 'Yes, within the past 12 months' in 2013 and 'No' in 2017, or (2) 'Yes, within the past 12 months' or 'Yes, but previously than within the past 12 months' in 2013 and 'No' in 2017.

Results The inconsistency percentages varied across CAM therapies. The highest levels of inconsistency for CAM use within the past 12 months were observed for nutritional counselling $(64.9 \%)$ and faith healing and/ or clairvoyance $(36.4 \%)$. The lowest proportion of inconsistent responses was observed for acupuncture (18.3\%). Overall, the same pattern was observed for lifetime CAM use.

Conclusions The results highlight the difficulty in obtaining reliable prevalence estimates on the use of CAM in the general population. Future studies should take these findings into account when interpreting similar analyses.

\section{BACKGROUND}

During the last decades, an increase in the use and acceptance of complementary and alternative medicine (CAM) has been
Strengths and limitations of this study

- No previous study has investigated the inconsistencies in self-reported use of complementary and alternative medicine (CAM) therapies.

Due to the study design, findings ca be generalised to the adult general population.

- The inclusion of six different CAM therapies makes it possible to compare inconsistencies in response patterns across the included therapies.

- Variations in specificity and number of CAM therapies within the CAM therapy response categories may affect comparability across CAM therapies.

- Lost to follow-up during the study period may, to some extent, compromise the validity of inconsistency estimates.

observed in Western countries. ${ }^{1-3}$ In Europe, the most commonly used CAM therapies are herbal medicine, homeopathy, chiropractic, acupuncture, reflexology and massage (for definitions of specific CAM therapies, please see reference 4) and it is estimated that up to $86 \%$ of the general population in Europe use CAM each year. ${ }^{5-8}$ However, comparing prevalence rates of CAM use across countries is very difficult since there is a large variation over time and between countries in which therapies that are considered to be conventional therapies or CAM therapies. ${ }^{9}$

The Danish healthcare system is universal and based on the principle that access to conventional healthcare is equal and free of charge for all citizens. ${ }^{10}$ However, according to a Danish survey, ${ }^{11}$ around $80 \%$ of the population are interested in using one or more types of CAM, whereas $51 \%$ believe that CAM can be just as effective as treatment offered by the conventional healthcare system. Also, more than half of the population fully or partly agree that they would be interested in 
combining CAM with conventional medical treatment if they got ill. ${ }^{11}$ Based on these findings, we consider the Danish population to be rather open to the potential health benefits embedded in CAM, and if diagnosed with a disease, preferably in combination with conventional healthcare.

CAM is typically used to complement biomedical care $^{1213}$ and for relaxation or improvement in subjective well-being. ${ }^{5}{ }^{14}$ Sociodemographic analyses find that CAM users are more likely to be females, middle-aged and have a higher education. ${ }^{5615}$ According to systematic reviews, a wide variety of health conditions is associated with CAM use with the most common being musculoskeletal problems,${ }^{5}$ back problems, depression, insomnia, severe headache or migraines, and stomach or intestinal illnesses. ${ }^{6}$

The use of CAM in the general population is typically estimated by means of survey data. ${ }^{513}{ }^{16}$ Such surveys rely greatly on the accuracy of the respondents' recall. Thus, the importance of accurate long-term memory is even more pronounced when the respondent is asked about lifetime use of CAM. Other factors that may affect data reliability include the respondent's motivation to provide truthful information on CAM. Reliable and accurate data is an important and valuable tool for various stakeholders and policy makers in order to monitor health behaviour in the population, evaluate the effectiveness of existing policies, and make or justify decisions. Also, it is well known that CAM is often used by specific populations, that is, those suffering from diseases such as cancer, ${ }^{17-19}$ diabetes, ${ }^{20-22}$ coronary heart disease ${ }^{23-25}$ and mental illness. ${ }^{26} 27$ As these specific populations often receive biomedical treatment as well, data on CAM use may serve as an important basis for determining if there are compromising or beneficial effects on disease progression if conventional and alternative treatment are used simultaneously.

The reliability of self-reported data on CAM use in the population can be examined by exploring their consistency over time, although consistency does not necessarily guarantee reliability. To our knowledge, no previous study has examined the consistency in self-reported CAM use over time. Previous studies have, however, carried out similar consistency analyses for other health-related indicators, for example, illicit drug use, ${ }^{28} 29$ smoking ${ }^{30} 31$ and specific health conditions. ${ }^{32}{ }^{33}$ Results from these studies indicate that inconsistency percentages are often surprisingly high. Furthermore, concerns have been raised about the validity of self-reported healthcare utilisation. ${ }^{34}$ Thus, the aim of this study was to examine inconsistencies in the use of various CAM therapies using longitudinal data from two survey waves conducted 4 years apart.

\section{METHODS}

Data were derived from the Danish Health and Morbidity Surveys, which are nationally representative health surveys that have been carried out regularly since $1987 .{ }^{35}$ The overall aim of the surveys is to describe the status and trends in health and morbidity in the general adult population in Denmark and factors that may influence health status. In this study, we use data from the two most recent survey waves in 2013 and 2017. In 2013, a random sample of 25000 adults ( $\geq 16$ years) was drawn from the Danish Civil Registration System (in which each citizen with an official residence in Denmark is registered with a unique personal registration number). ${ }^{36}$ All randomly selected individuals were sent a postal questionnaire in 2013, but throughout the data collection period it was also possible to complete an identical web questionnaire. A total of 14265 individuals completed the self-administered questionnaire in 2013, corresponding a response rate of $57 \%$. In 2017, a nationally representative subsample of 3147 respondents were reinvited to participate in the next survey wave in 2017 using the same mode of administration as in 2013. As 161 individuals were lost to follow-up due to death or emigration, and 689 were lost due to non-response, the total sample size in this study included 2297 individuals. The data collection period was between February and mid-May in both survey waves. Furthermore, in both survey waves, CAM use was assessed by asking the respondents the following question: 'Have you ever been treated by therapists outside the general health services

Table 1 Baseline characteristics of the study population $(n=2297)$

\begin{tabular}{|c|c|c|c|c|c|c|}
\hline CAM therapy & $\begin{array}{l}\text { Use within the past } \\
12 \text { months (\%) }\end{array}$ & $\begin{array}{l}\text { Women } \\
(\%)^{\star}\end{array}$ & $\begin{array}{l}\text { Mean age } \\
\text { (SD)* }\end{array}$ & $\begin{array}{l}\text { Lifetime use } \\
(\%) \dagger\end{array}$ & $\begin{array}{l}\text { Women } \\
(\%)^{*}\end{array}$ & $\begin{array}{l}\text { Mean age } \\
(\mathrm{SD})^{\star}\end{array}$ \\
\hline Acupuncture & 8.4 & 67.2 & $50.1(15.7)$ & 28.2 & 65.6 & $52.0(14.7)$ \\
\hline Faith healing and/or clairvoyance & 2.6 & 81.8 & $52.8(14.6)$ & 8.3 & 78.5 & $49.8(14.1)$ \\
\hline Nutritional counselling & 1.7 & 73.0 & $53.5(16.8)$ & 6.0 & 76.7 & $52.0(15.7)$ \\
\hline Reflexology & 5.3 & 69.9 & $52.7(14.6)$ & 24.6 & 70.3 & $52.4(14.1)$ \\
\hline
\end{tabular}

*Among individuals who have used CAM before baseline.

†Lifetime use of CAM.

CAM, complementary and alternative medicine. 
and, for example, used any of the following providers or forms of treatment?'. The following providers or forms of therapies were included: acupuncture; craniosacral therapy; faith healing and/or clairvoyance; nutritional counselling (individualised nutrition counselling); massage, osteopathy or other manipulative therapies; reflexology (of which foot reflexology is the frequently used form in Denmark). Possible response categories for each CAM therapy were 'Yes, within the past 12 months', 'Yes, but previously than within the past 12 months' and 'No'. The respondents were also asked about their use of other CAM therapies (eg, applied kinesiology and homeopathy), but these therapies were excluded from this study due to low prevalence estimates. In this study, two types of inconsistencies were examined for each CAM therapy. The first type of inconsistent response was defined as when a respondent answered 'Yes, within the past 12 months' to a specific CAM therapy in the survey wave in 2013, but then answered 'No' to the same CAM therapy in the 2017 survey wave. The second type of inconsistent response was defined as when a respondent answered 'Yes, within the past 12 months' or 'Yes, but previously than within the past 12 months' to a specific CAM therapy in the survey wave 2013 (ie, lifetime use), but then answered 'No' to the same CAM therapy in 2017 survey wave.

Information on sex and age was obtained from the Danish Civil Registration System. ${ }^{36}$ Educational level was the self-reported highest completed level of education.

\section{Patient and public involvement}

Patients or the public were not involved in the design, or conduct, or reporting, or dissemination plans of our research.

\section{Statistical analysis}

The inconsistencies are presented as percentages with 95\% CIs, which were calculated using the Wilson score method.

Multiple logistic regression models were used to identify factors associated with inconsistent response among lifetime use of acupuncture, massage, osteopathy or other manipulative therapies and reflexology, respectively. The results are presented as odds ratios (OR) with 95\% CIs. The first model was adjusted for sex, age and use of CAM practitioner within the past 12 months reported at baseline. The second model was further adjusted for combined school and vocational education (these analyses were restricted to individuals aged 25 years or older) since most have completed their education by this point. Statistical analyses were performed using SAS V.9.4.

\section{RESULTS}

The baseline characteristics of the respondents completing the self-administered questionnaire in both 2013 and 2017 are presented in table 1. In all, 21.2\% had used massage, osteopathy or other manipulative 
therapies within the past 12 months. Acupuncture and reflexology had the second and third highest prevalence estimate, respectively, at $8.4 \%$ and $5.3 \%$. The table also shows that CAM users were more likely to be women than men for all six CAM therapies. The mean age of CAM users varied between 48.1 years (massage, osteopathy or other manipulative therapies) and 53.5 years (nutritional counselling). Furthermore, prevalence rates of lifetime use of CAM therapies varied between $36.4 \%$ (massage, osteopathy or other manipulative therapies) and nutritional counselling $(6.0 \%)$.

In table 2, the inconsistency percentages for each CAM therapy is presented for CAM use within the past 12 months and for lifetime use (ie, ever use) of CAM, respectively. The inconsistency percentages vary greatly across CAM therapies. In all, $64.9 \%$ of the individuals who in the baseline survey (in 2013) reported that they had used nutritional counselling within the past 12 months answered that they had never used this therapy in the subsequent survey wave (in 2017). A high proportion of inconsistent responses was also observed for faith healing and/or clairvoyance $(36.4 \%)$. The lowest inconsistency percentages were observed for acupuncture (18.3\%) and massage, osteopathy or other manipulative therapies $(22.9 \%)$. Table 2 also shows that $62.0 \%$ of the individuals who in the baseline survey (in 2013) reported that they had ever used nutritional counselling (ie, lifetime use) answered that they had never used this therapy in the subsequent survey wave (in 2017). The inconsistency pattern for lifetime use of CAM is similar to that seen for CAM use within the past 12 months.

Table 3 shows the inconsistency percentages for lifetime use of CAM according to sex. The inconsistency percentages were significantly higher among men than women for two CAM therapies (massage, osteopathy or other manipulative therapies, and reflexology). The percentages were higher among men for three out of the four other CAM therapies, although the results were not statistically significant.

The results from the multiple logistic regression analyses revealed that the predictors that were associated with inconsistent response varied by lifetime use of CAM therapies (table 4). For example, male sex was associated with increased odds of inconsistent response (in 2017) for massage, osteopathy or other manipulative therapies
(OR $1.47,95 \%$ CI 1.06 to 2.04 ) and reflexology (OR $1.94,95 \%$ CI 1.29 to 2.93 ), respectively. However, we found no significant association between sex and inconsistent response for individuals who reported lifetime use of acupuncture. Age was an independent predictor of all three outcomes, but the pattern of associations differed slightly by CAM therapy. Furthermore, table 4 shows that individuals who had not used massage, osteopathy, or other manipulative therapies within the past 12 months had 2.38 (95\% CI 1.73 to 3.28) times higher odds of reporting never having used this CAM therapy in the subsequent wave than individuals who had used it within the past year. Interestingly, no significant associations were observed between use within the past year and the two other CAM therapies. Finally, the results of the logistic regression models indicated that an inconsistent response was associated with a lower level of education among all three CAM therapies, however, only borderline significant for lifetime use of massage, osteopathy or other manipulative therapies.

\section{DISCUSSION}

This study examined the inconsistency in self-reported use of various CAM therapies using longitudinal data from two survey waves conducted 4 years apart. To our knowledge, no previous studies have examined this matter. We found high levels of inconsistencies for all CAM therapies. The highest proportion of inconsistent responses was observed for nutritional counselling and the lowest for acupuncture. Although there are no comparable studies, the response patterns are, to some extent, similar to those found for other health-related outcomes. For example, it has been shown that response consistency over time depends on the severity and type of health condition. ${ }^{32} 33$ Applying a similar logic to the findings from this study, one could argue that the more 'invasive' the CAM therapy, the lower inconsistency percentages. This could probably be explained by the fact that people tend to remember experiences that create a lasting memory, for example, pain due to acupuncture needles inserted into the body as opposed to for example, increased well-being due to massage. Moreover, acupuncture is a very well-defined CAM therapy, whereas for example, nutritional counselling, the CAM therapy exhibiting the highest proportion

\begin{tabular}{|c|c|c|c|}
\hline CAM therapy & Men & Women & $P$ value \\
\hline Acupuncture & 23.1 (17.9 to 29.3$)$ & 21.7 (17.9 to 26.0$)$ & 0.691 \\
\hline Craniosacral therapy & 25.8 (13.7 to 43.3$)$ & 28.9 (21.8 to 37.3$)$ & 0.731 \\
\hline Faith healing and/or clairvoyance & 36.8 (23.4 to 52.7$)$ & 30.9 (23.9 to 39.1$)$ & 0.490 \\
\hline Nutritional counselling & 73.3 (55.6 to 85.8$)$ & 58.6 (48.7 to 67.8$)$ & 0.145 \\
\hline Massage, osteopathy or other manipulative therapies & 36.1 (30.8 to 41.8$)$ & 27.3 (23.6 to 31.5$)$ & 0.010 \\
\hline Reflexology & 37.0 (29.8 to 44.7$)$ & 22.9 (18.9 to 27.5$)$ & $<0.001$ \\
\hline
\end{tabular}

CAM, complementary and alternative medicine. 


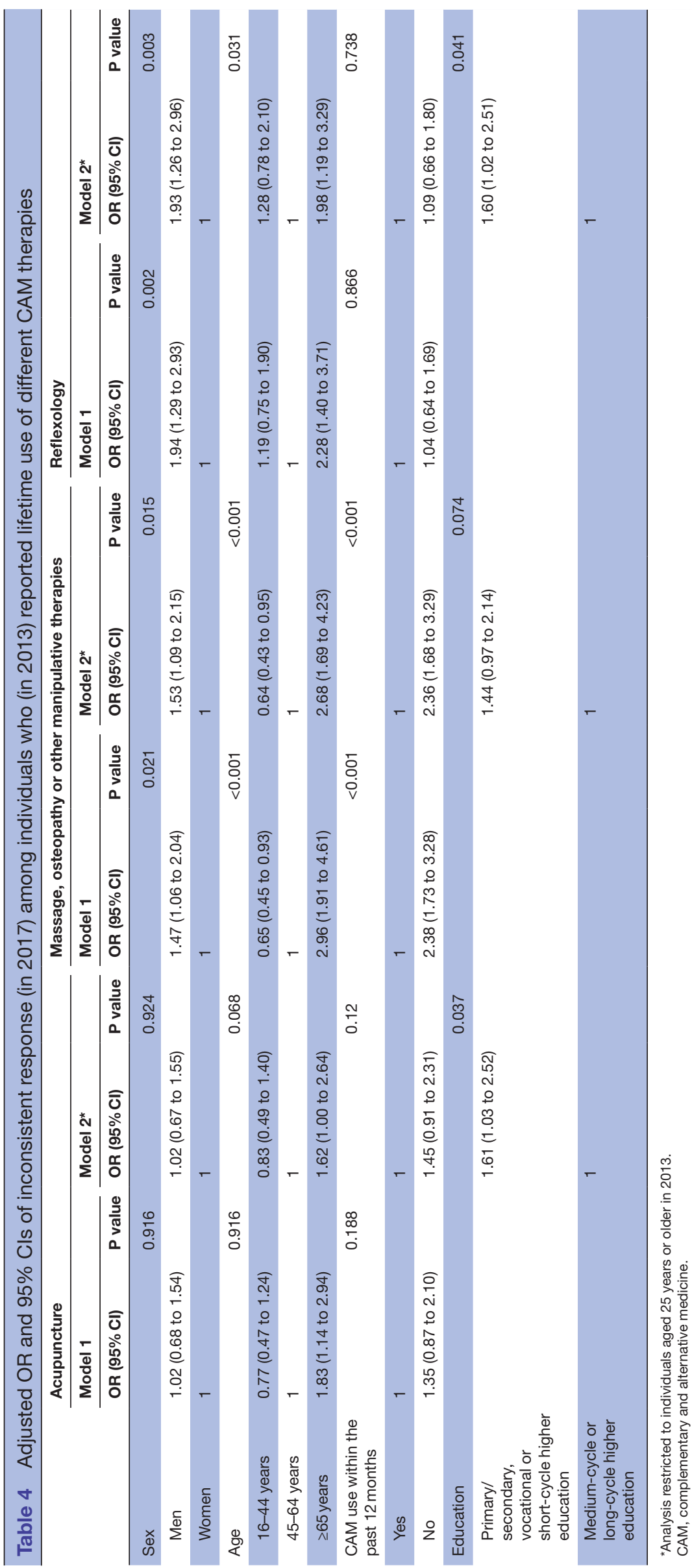

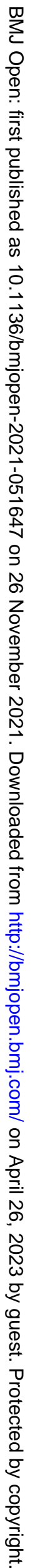


of inconsistent responses, is a quite vague definition, which may have introduced some uncertainties in the respondents. For example, is it considered nutritional counselling or not if a friend recommends that you eat healthier or a TV show or a book inspire you to do so? Or should the nutritional counselling have been provided by a certified dietitian to be considered nutritional counselling? Another factor that may blur the memory of nutritional counselling is related to the fact that information on diet and nutrition is often provided from other sources than educated counsellors or dietitians, that is, by the media. Accordingly, the respondent may simply forget the origin of the nutritional advice, thus resulting in response inconsistencies over time.

Our results revealed some interesting sociodemographic patterns in relation to CAM use, which are in line with the findings from previous studies. For example, all CAM therapies are more frequently used by women than by men and with a mean age by users of around 50 years. ${ }^{5615}$ Inconsistent responses were related to both sex, age, educational level and use of CAM therapy within the past year, depending on the type of CAM therapy. We also found that use of CAM therapies was more prevalent among women than among men. This finding is in keeping with a recent study based on data from 21 European countries. ${ }^{8}$ Thus, the sex-dependent inconsistent responses reported in this study could probably, at least partly, be explained by men being more likely to forget their previous use because of their low(er) use. Women, on the other hand, report higher rates of unmet needs, more healthcare utilisation and poorer health, which may make them more likely to actively seek for alternative treatment options, that is, CAM. ${ }^{8}$ These motivations may cause women to better remember their previous CAM use than men.

The main strength of our study is that no previous study has investigated the inconsistencies in self-reported use of CAM therapies, which makes the study its own of its kind. Also, an important strength of the study is that the findings can be generalised to the adult general population in relation to the self-report inconsistencies in the use of CAM therapies. Moreover, the long list of various CAM therapies makes it possible to compare inconsistencies in response patterns across the included therapies.

This study has some potential limitations that need to be addressed. First, the included CAM therapy response categories differed in, for example, their specificity and the number of therapies. For example, the response category 'Acupuncture' includes only one specific CAM therapy, whereas the response category 'Massage, osteopathy or other manipulative therapies' or 'Faith healing and/or clairvoyance' includes more and less well-defined CAM therapies. This makes it somewhat difficult to compare specific CAM therapies. For the CAM therapy 'Nutritional counselling', it was not specified whether only verbal counselling was included, or if also nutritional supplements were provided. Moreover, lost to follow-up during the study period may, to some extent, have compromised the validity of inconsistency estimates. Lastly, a general source of bias when examining self-reported use of CAM is related to societal trends in and definitions of which therapies that are considered conventional therapies or CAM therapies, respectively. Acupuncture, for example, which was defined as a CAM therapy in our study may also be considered a conventional therapy by some respondents as it may sometimes be offered to specific patient groups in public hospital settings. Such grey zone areas concerning the constitution and definition of CAM therapies may have considerable implications for survey results and, accordingly, inconsistency estimates.

Recommendations from previous research in other scientific areas exploring inconsistencies in self-reported health-related behaviour highlight the importance of using repeated measures or ask about the frequency of the behaviour of interest. ${ }^{29}$ In this way, one may find out that the majority of inconsistent responses occur in respondents who only engage in that specific behaviour, for example, used nutritional counselling, once or twice in their life. Such responses may then not be considered relevant from a public health perspective. Similar recommendations could be formulated based on the findings from this study. Another recommendation could be to primarily assess the use of CAM in face-to-face and telephone interview surveys where an interviewer is present to explain the question/therapies in detail and probe for more information if the respondent is uncertain about a certain question. Lastly, it is recommended for future surveys on CAM use to include clear definitions of all types of CAM use and define CAM in general in all surveys.

\section{CONCLUSIONS}

In conclusion, the high proportions of inconsistent responses demonstrated in this study highlight the difficulty in obtaining reliable prevalence estimates on the use of CAM in the general population. Although inconsistencies were found for all included CAM therapies, large inconsistency variations across CAM therapies were demonstrated with the highest proportion of inconsistent response for nutritional counselling, the lowest for acupuncture. Our results provide new insight into possible methodological challenges in health surveys which include questions on CAM use that need to be addressed and taken into account when interpreting findings from similar studies and planning future studies. Indeed, our results underscore the importance of including clear and well-defined questions when asking about CAM in surveys.

Acknowledgements We gratefully acknowledge all respondents who participated in the surveys.

Contributors The study was conceptualised by both authors. HARJ wrote the first draft of the manuscript; $\mathrm{OE}$ carried out the statistical analyses. Both authors critically reviewed and approved the final version of the manuscript. As guarantor, $\mathrm{OE}$ accepts full responsibility for the finished work and the conduct of the study. $\mathrm{OE}$ had access to the data and controlled the decision to publish.

Funding The authors have not declared a specific grant for this research from any funding agency in the public, commercial or not-for-profit sectors. 
Competing interests None declared.

Patient and public involvement Patients and/or the public were not involved in the design, or conduct, or reporting, or dissemination plans of this research.

Patient consent for publication Not applicable.

Ethics approval The study was approved by SDU RIO (ID 10.899). SDU RIO examines and approves all scientific and statistical projects at the University of Southern Denmark according to the Danish Data Protection Regulation. Ethical approval is not required for surveys according to Danish legislation.

Provenance and peer review Not commissioned; externally peer reviewed.

Data availability statement Data are available on reasonable request. The datasets used and/or analysed for the present study are available from the corresponding author on reasonable request. The authors encourage collaboration, and that data are used by other researchers.

Open access This is an open access article distributed in accordance with the Creative Commons Attribution Non Commercial (CC BY-NC 4.0) license, which permits others to distribute, remix, adapt, build upon this work non-commercially, and license their derivative works on different terms, provided the original work is properly cited, appropriate credit is given, any changes made indicated, and the use is non-commercial. See: http://creativecommons.org/licenses/by-nc/4.0/.

\section{ORCID iDs}

Heidi Amalie Rosendahl Jensen http://orcid.org/0000-0001-7171-7429

Ola Ekholm http://orcid.org/0000-0002-5563-7098

\section{REFERENCES}

1 Clarke T, Black LI, Stussman BJ, et al. Trends in the use of complementary health approaches among adults: United States, 2002-2012. Natl Health Stat Report 2015;79:1-16.

2 Fischer FH, Lewith G, Witt CM, et al. High prevalence but limited evidence in complementary and alternative medicine: guidelines for future research. BMC Complement Altern Med 2014;14:46.

3 Ernst E, White A. The BBC survey of complementary medicine use in the UK. Complement Ther Med 2000;8:32-6.

4 Institute of Medicine. Complementary and alternative medicine in the United States. Washington DC: The National Academies Press, 2005.

5 Eardley S, Bishop FL, Prescott P, et al. A systematic literature review of complementary and alternative medicine prevalence in EU. Forsch Komplementmed 2012;19:18-28.

6 Frass M, Strassl RP, Friehs $\mathrm{H}$, et al. Use and acceptance of complementary and alternative medicine among the general population and medical personnel: a systematic review. Ochsner J 2012;12:45-56.

7 Kemppainen LM, Kemppainen TT, Reippainen JA, et al. Use of complementary and alternative medicine in Europe: health-related and sociodemographic determinants. Scand J Public Health 2018;46:448-55.

8 Fjær EL, Landet ER, McNamara CL, et al. The use of complementary and alternative medicine (cam) in Europe. BMC Complement Med Ther 2020;20:108.

9 Hanssen B, Grimsgaard S, Launsø L, et al. Use of complementary and alternative medicine in the Scandinavian countries. Scand J Prim Health Care 2005;23:57-62.

10 Health Care Denmark \& the Danish Ministry of Health. Health care in Denmark - an overview. Copenhagen: the Danish Ministry of Health, 2017.

11 Mandag Morgen \& TrygFonden. Sundhedsvæsenet - ifølge danskerne. København: Mandag Morgen; Virum: TrygFonden, 2016.

12 Macartney JI, Wahlberg A. The problem of complementary and alternative medicine use today: eyes half closed? Qual Health Res 2014;24:114-23.

13 Shmueli A, Igudin I, Shuval J. Change and stability: use of complementary and alternative medicine in Israel: 1993, 2000 and 2007. Eur J Public Health 2011;21:254-9.
14 Nissen N, Schunder-Tatzber S, Weidenhammer W, et al. What attitudes and needs do citizens in Europe have in relation to complementary and alternative medicine? Forsch Komplementmed 2012;19:9-17.

15 Harris PE, Cooper KL, Relton C, et al. Prevalence of complementary and alternative medicine (CAM) use by the general population: a systematic review and update. Int J Clin Pract 2012;66:924-39.

16 Posadzki P, Watson LK, Alotaibi A, et al. Prevalence of use of complementary and alternative medicine (CAM) by patients/ consumers in the UK: systematic review of surveys. Clin Med 2013;13:126-31.

17 Kathrin W, Henriksson R, Sharp L, et al. Cancer patients' use of complementary and alternative medicine in Sweden: a crosssectional study. BMC Complemen Altern Med 2019;19:62.

18 Lee S-H, Kim J-Y, Yeo S, et al. Meta-analysis of massage therapy on cancer pain. Integr Cancer Ther 2015;14:297-304.

19 Molassiotis A, Fernández-Ortega P, Pud D, et al. Use of complementary and alternative medicine in cancer patients: a European survey. Ann Oncol 2005;16:655-63.

20 Yang W-C, Chang CLT, Li C-R, et al. Complementary and alternative medicine for diabetes 2014. Evidence-Based Complementary and Alternative Medicine 2015;2015:1-2.

21 Birdee GS, Yeh G. Complementary and alternative medicine therapies for diabetes: a clinical review. Clin Diabetes 2010;28:147-55.

22 Dham S, Shah V, Hirsch S, et al. The role of complementary and alternative medicine in diabetes. Curr Diab Rep 2006;6:251-8.

23 Kristoffersen AE, Sirois FM, Stub T, et al. Prevalence and predictors of complementary and alternative medicine use among people with coronary heart disease or at risk for this in the sixth Troms $\varnothing$ study: a comparative analysis using protection motivation theory. BMC Complement Altern Med 2017;17:324.

24 Grant SJ, Bin YS, Kiat H, et al. The use of complementary and alternative medicine by people with cardiovascular disease: a systematic review. BMC Public Health 2012;12:299.

25 Rabito MJ, Kaye AD. Complementary and alternative medicine and cardiovascular disease: an evidence-based review. Evid Based Complement Alternat Med 2013;2013:1-8.

26 Fogarty S, Smith CA, Hay P. The role of complementary and alternative medicine in the treatment of eating disorders: a systematic review. Eat Behav 2016;21:179-88.

27 Barić H, Đorđević V, Cerovečki I, et al. Complementary and alternative medicine treatments for generalized anxiety disorder: systematic review and meta-analysis of randomized controlled trials. Adv Ther 2018:35:261-88.

28 Fendrich M, Rosenbaum DP. Recanting of substance use reports in a longitudinal prevention study. Drug Alcohol Depend 2003;70:241-53.

29 Jensen HAR, Karjalainen K, Juel K, et al. Consistency in adults' selfreported lifetime use of illicit drugs: a follow-up study over 13 years. J Stud Alcohol Drugs 2018;79:490-4.

30 Stanton CA, Papandonatos G, Lloyd-Richardson EE, et al. Consistency of self-reported smoking over a 6-year interval from adolescence to young adulthood. Addiction 2007;102:1831-9.

31 Kaestle CE. Age of smoking milestones: longitudinal inconsistencies and recanting. J Adolesc Health 2015;56:382-8.

32 Beckett M, Weinstein M, Goldman N, et al. Do health interview surveys yield reliable data on chronic illness among older respondents? Am J Epidemiol 2000;151:315-23.

33 Jensen HAR, Davidsen M, Christensen Al, et al. Inconsistencies in self-reported health conditions: results of a nationwide panel study. Int J Public Health 2019;64:1243-6.

34 Bhandari A, Wagner T. Self-reported utilization of health care services: improving measurement and accuracy. Med Care Res Rev 2006;63:217-35.

35 Jensen HAR, Ekholm O, Davidsen M, et al. The Danish health and morbidity surveys: study design and participant characteristics. BMC Med Res Methodol 2019;19:91.

36 Pedersen CB. The Danish civil registration system. Scand J Public Health 2011;39:22-5. 